\title{
Effect of Antiferromagnetic Anisotropy on Exchange Bias in a Single Composite Nanoparticle with Unconventional Antiferromagnetic-Ferromagnetic Core-Shell Morphology*
}

\author{
Yong $\mathrm{Hu}$, Yan Liu, Yan Qi, and $\mathrm{An} \mathrm{Du}^{\dagger}$ \\ College of Sciences, Northeastern University, Shenyang 110-819, China \\ (Received 14 September 2010; Accepted 9 February 2011; Published 5 March 2011)
}

\begin{abstract}
In recent years, it has been found that magnetic anisotropy in antiferromagnetic layers affects strongly exchange bias in bilayers or multilayers. However, research on magnetic nanoparticles or nanostructures is lacking. Therefore at low temperature after cooling under different fields, antiferromagnetic-anisotropy dependence of exchange bias in a single nanoparticle with unconventional antiferromagnetic (core)/ferromagnetic (shell) morphology is simulated by using a modified Monte Carlo Metropolis method, which has proved an efficient method to deal with the models with the consideration of the finite anisotropies. Common (negative) exchange bias always exists at low temperature after cooling under weak fields, independent of antiferromagnetic anisotropy. For strong cooling fields, nevertheless, positive exchange bias appears only when the antiferromagnetic anisotropy is above a critical value. The numerical results are interpreted well by means of the microscopic spin configurations of nanoparticle. The systematic study on the nanoparticle with such a special morphology will illuminate a way to solve the exchange bias puzzles.

[DOI: 10.1380/ejssnt.2011.67]
\end{abstract}

Keywords: Nano-particles; Nucleation; Exchange bias; Antiferromagnetic anisotropy; Microscopic spin configurations; Metastable states; Contacts; Monte Carlo simulations

\section{INTRODUCTION}

Understanding the magnetic properties of nanometer scale particles is a central issue in magnetic materials. Magnetic nanoparticles themselves are used as the active components of ferrofluids, recording tapes, flexible disk recording media, as well as biomedical materials and catalysts. Furthermore, assemblies of nano-scale magnetic grains make up hard disk recording media, permanent magnets, and nanocrystalline soft materials. These diverse technological applications are the focuses of scientific research. Magnetic nanoparticles are also used as research tools in areas of materials physics, geology, biology, and medicine [1]. Due to their reduced dimensions, nanoparticles display peculiar magnetic and transport properties which are not present in the bulk materials as a consequence of the interplay between intrinsic properties arising from finite-size effects and collective effects due to different kinds of interparticle interactions. A direct consequence of the finite size in nanoparticles is the superparamagnetism, which is a drawback for magnetic recording applications because it causes thermal destabilization of the recording units [2]. A phenomenon known as exchange bias (EB), which is attributed to the exchange interaction at the interface between ferromagnetic (FM) and antiferromagnetic (AFM) components, may be a way to beat the superparamagnetic limit [3].

Fine particle was the first type of system where exchange bias was observed. Meiklejohn and Bean found that hysteresis loops shifted along the magnetic field axis in the partially oxidized Co particles [4]. EB has been extensively studied for the past ten years, partly because of its application in ultrahigh-density magnetic record-

\footnotetext{
*This paper was presented at the 6th International Workshop on Nano-scale Spectroscopy and Nanotechnology (NSS-6), Kobe University Centennial Hall, Kobe, Japan, 25-29 October, 2010.

${ }^{\dagger}$ Corresponding author: duanneu@126.com
}

ing, giant magnetoresistance (GMR), and "spin valve" devices [5]. In 1996 Nogués et al. firstly observed a positive EB field $\left(H_{E}\right)$ in $\mathrm{FeF}_{2} / \mathrm{Fe}$ bilayers with AFM interfacial coupling [6]. Since then, the phenomena of positive $H_{E}$ have also been observed in both ferrimagnetic (FI)/FI and FM/FI bilayers $[7,8]$. Besides positive $H_{E}$, many interesting and striking properties, such as vertical loop shifts [9] and asymmetrical shaped loops [10-12], have been heavily revealed and studied in several types of composite nanoparticle systems, including the ones with traditional [13] or inverted core-shell structures [14, 15].

Experimentally and theoretically, the antiferromagnets play a key role in the FM/AFM exchange-biased systems [16]. EB is usually affected by the AFM defects [1719] and thicknesses [20-22] strongly. Additionally, Mauri et al. [23] used an in situ Kerr rotation measurement to estimate the magnetic anisotropy of $\mathrm{MnFe}$ in exchangebiased $\mathrm{Mn}_{50} \mathrm{Fe}_{50} / \mathrm{Ni}_{80} \mathrm{Fe}_{20}$ bilayers. Recently, Mitsumata et al. [24] also verified that the cubic anisotropy in the disorder AFM $\gamma$-phase Mn alloys is closely associated with the exchange bias characteristics as well as the shape of torque curves of the FM/AFM bilayers. However, detailed research on AFM anisotropy dependence of EB in either experiment or theory is still lacking. Moreover, very recently many Mn-base "inverse" core/shell nanoparticles have been synthesized and a number of novel magnetic properties are obtained [15, 25-27]. In our previous work, we have studied the effects of cooling field and particle shape on EB and asymmetric magnetization reversal in spherical or egg-shaped inverted core/shell nanoparticles $[28,29]$ as well as the effects of AFM exchange coupling and anisotropy on EB in inverted core/matrix systems [30]. A sign-reversal behavior of EB may be observed and the phenomenon is very intriguing. Therefore in this paper, the role of AFM anisotropy in establishing the EB properties in a single composite nanoparticle with AFM interfacial coupling and inverted AFM (core)/FM (shell) morphology is studied by performing a modified Monte Carlo (MC) Metropolis method and the phenomena are interpreted well by means of the microscopic spin 
configurations of nanoparticle around the coercive fields of hysteresis loops at low temperature.

\section{MODEL AND MONTE CARLO METHOD}

The simulated composite nanoparticle in a spherical shape (total radius $R=10 a$ ) consists of an AFM core
$\left(R_{C}=6 a\right)$, soft FM shells $\left(t_{S H}=3 a\right)$, and an outer hard FM skin $\left(t_{S K}=1 a\right)$ where spins have reduced coordination numbers with respect to the interior. $a$ is the lattice constant and taken as $a=0.3 \mathrm{~nm}$, thus such a core/shell nanoparticle corresponds to typical scaled dimension approximately $R=3.0 \mathrm{~nm}$.

The Hamiltonian of system under an external magnetic field is given by

$$
\begin{aligned}
\mathcal{H}= & -J_{C} \sum_{\langle i, j \in A F M c o r e\rangle} \mathbf{S}_{i} \cdot \mathbf{S}_{j}-J_{I} \sum_{\langle i \in A F M c o r e, j \in F M \text { shell }\rangle} \mathbf{S}_{i} \cdot \mathbf{S}_{j}-J_{S} \sum_{\langle i, j \in F M \text { shell }(\text { skin })\rangle} \mathbf{S}_{i} \cdot \mathbf{S}_{j} \\
& -\sum_{i \in A F M c o r e} K_{C}\left(\mathbf{S}_{i} \cdot \widehat{\mathbf{e}}_{i}\right)^{2}-\sum_{i \in F M \text { shell }} K_{S H}\left(\mathbf{S}_{i} \cdot \widehat{\mathbf{e}}_{i}\right)^{2}-\sum_{i \in F M \text { skin }} K_{S K}\left(\mathbf{S}_{i} \cdot \mathbf{n}_{i}\right)^{2} \\
& -\mathbf{H} \cdot \sum_{i} \mathbf{S}_{i}
\end{aligned}
$$

where $\mathbf{S}_{i}$ is the classical Heisenberg spin of unit magnitude placed at the nodes of a simple cubic lattice and $\widehat{\mathbf{e}}_{i}$ is the unit vector in the direction of the easy axis at site $i$. The angular brackets in the sums represent a summation over the nearest neighbors only. The first three terms contain the energy contributions of exchange coupling, in which $J_{S}=1.0, J_{I}=-0.6 J_{S}$, and $J_{C}=-0.5 J_{S}$ denote the exchange coupling constants reduced by $J_{S}$ in the FM shell (skin), the AFM/FM interface, and the AFM core, respectively. They just fix the Curie temperature of the ferromagnet to $T_{C}=1.5$ and the Néel temperature of the antiferromagnet to $T_{N}=0.75$, a value lower than $T_{C}$ as is the case in most oxides with respect to their native materials. Likewise, the anisotropy energies are considered in the next three terms, where $K_{S H}=0.002 J_{S}$ denotes the uniaxial anisotropy constant of spin in the FM shell just in order to obtain the obvious hysteresis loops and $K_{S K}=0.2 J_{S}$ denotes the one in the FM skin which is two orders of magnitude larger than $K_{S H}$, while the uniaxial anisotropy constant of spin in the AFM core $K_{C}$ is varied and has to be higher than that in the FM shell as required to pin the AFM spins during the hysteresis loops at low temperature so that EB is observed. Finally, the last term gives the Zeeman energy; $\mathbf{H}$ is supposed to be along the $z$ axis. The long-range dipolar interactions are not taken into account due to the small size of nanoparticle. In experiments, Inderhees et al. have found that the core/shell interface may be highly crystalline and directional and have the low level of disordered characteristic [31]. Additionally, the surface atoms of nanoparticle have a reduced symmetry where the axis of the electric field gradient (the leading crystal field term $A_{2}^{0}$ ) tends to lie perpendicular to the surface, leading to the anisotropy which is perpendicular to the surface or else lies in a plane parallel to the surface in principle $[32,33]$. It is the origin of the surface anisotropy, which was first discussed by Néel [34]. Therefore, the edge effect of nanoparticle must be considered individually in this paper due to the unconventional morphology. As a result, the anisotropy axes of spin in the AFM core and soft FM shell are supposed to be along the $z$ axis, whereas on the surface of nanoparti- cle the locally radial easy axes of spin are introduced to mimic the surface anisotropy. The model of nanoparticle is illustrated as shown in Fig. 1. $H$ and $H_{E}$ are given in units of $J_{S} / g \mu_{B}, T$ in units of $J_{S} / k_{B}$, and $K_{C}$ in units of $J_{S}$.

The aforementioned model is simulated by performing the MC method with a modified Metropolis algorithm, in which the flipping probability depends not only on the energies of initial and final states but also on the saddlepoint energy between two states. If the saddle-point energy is higher than the energy of final state, the flipping probability depends on the energy difference between the initial state and the saddle point [35]. Our protocol to simulate the field-cooling process is as follows. Under a magnetic field $\left(H_{F C}\right)$, the system with a disordered spin configuration is cooled from $T=2.0$, which is above $T_{C}$, to a target low temperature $\left(k_{B} T / J_{S}=0.01\right)$, which is

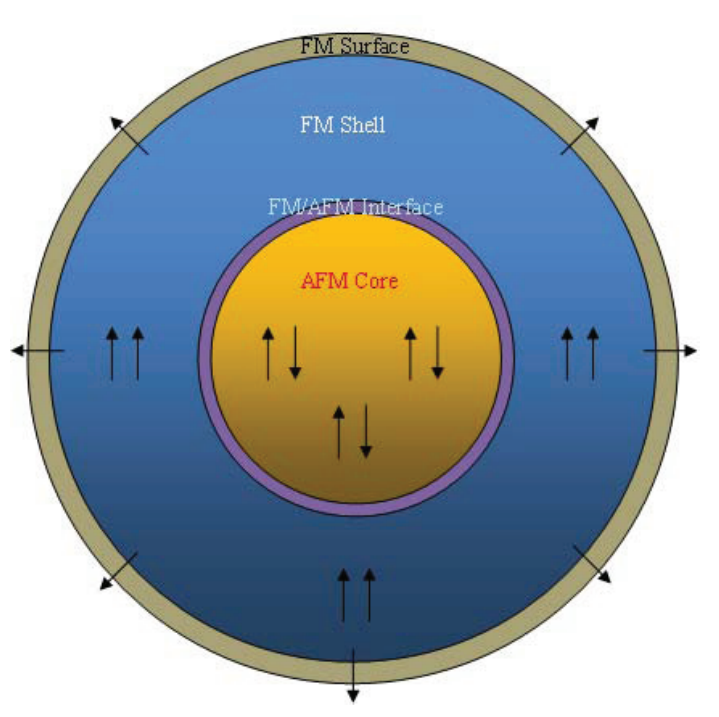

FIG. 1: Schematic illustration of the composite nanoparticle with inverted antiferromagnetic (core)/ferromagnetic (shell) morphology. The arrows represent the typical spin orientations in the individual parts. 

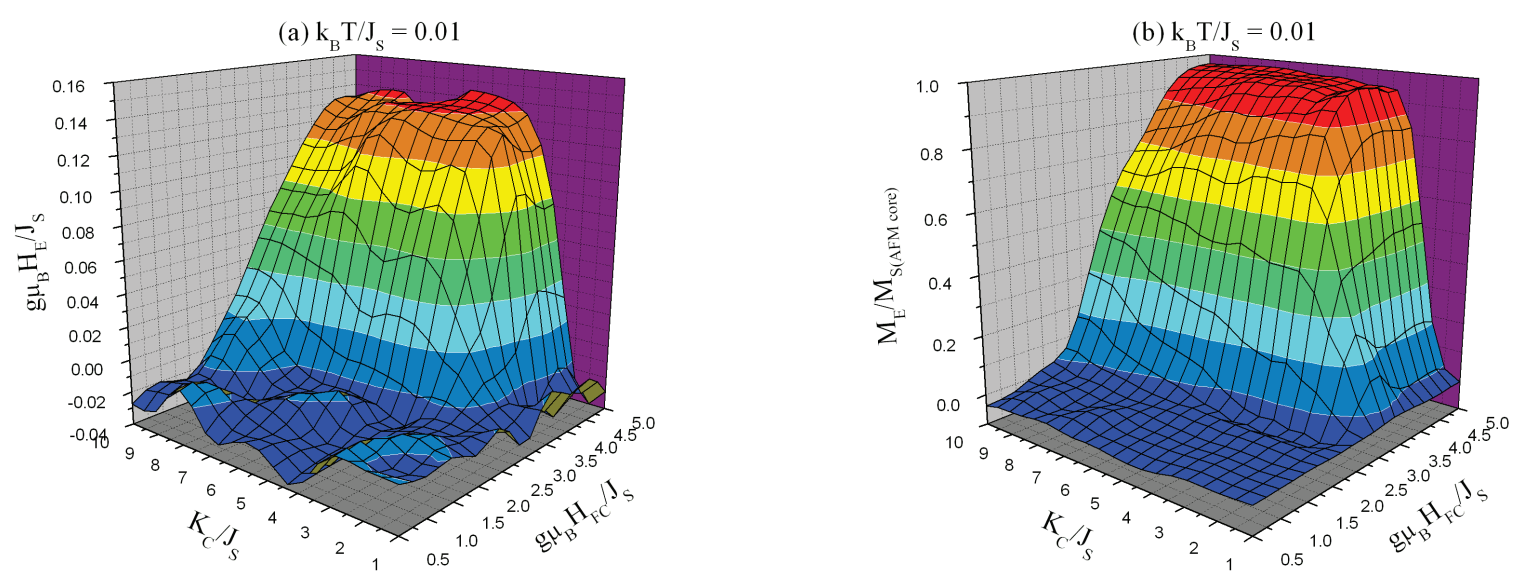

FIG. 2: (a) Exchange bias field $H_{E}$ and (b) normalized magnetization vertical shift $M_{E} / M_{S(A F M c o r e)}$ as functions of anisotropy of spin in the antiferromagnetic core $K_{C}$ and cooling field $H_{F C}$ at $k_{B} T / J_{S}=0.01 . M_{S(A F M c o r e)}$ is the saturated magnetization of antiferromagnetic core.

far below the blocking temperature of super-paramagnetic state and $T_{N}$ absolutely [36]. Then at $k_{B} T / J_{S}=0.01$ the hysteresis loop was recorded from $H=0.4\left(+H_{\max }\right)$ to $-0.4\left(-H_{\max }\right)$ and afterwards raised again up to the initial value. Actually, the range of magnetic field is responsible for EB strongly. Sort et al. [37] have presented that $H_{E}$ is roughly constant in a relatively broad $H_{\max }$ range, and progressively decreases up to zero for stronger $H_{\max }$. Whereas under weak $H_{\max }$, someone has ever discussed if samples measured at low temperature are unsaturated, unreliable results may be obtained in experiments [38-41]. Therefore, $H_{\max }$ in the present paper is chosen not too strong but also must guarantee the saturation of the FM shell in order to avoid the minor loops and extract the available EB parameters. Furthermore in simulations, we perform typically $580000 \mathrm{MC}$ steps per spin for a complete magnetization curve in the cooling process and 2820000 MC steps per spin for a complete hysteresis loop at low temperature. In other words, $20000 \mathrm{MC}$ steps per spin are used at every temperature value during cooling and another $20000 \mathrm{MC}$ steps per spin are performed at every field value during the hysteresis loops; the thermal average of magnetization is performed with $10000 \mathrm{MC}$ steps after the initial $10000 \mathrm{MC}$ steps, which were discarded for thermalization.

\section{RESULTS AND DISCUSSION}

The field-cooled hysteresis loops are shifted along the field and magnetization axes simultaneously. The shift may be quantified through the $\mathrm{EB}$ parameter $H_{E}=$ $-\left(H_{R I G H T}+H_{L E F T}\right) / 2, H_{R I G H T}$ and $H_{L E F T}$ being the points where the loop of FM shell intersects the field axis. Moreover, the vertical shift of hysteresis loop is characterized by $M_{E}=\left(M\left(+H_{\max }\right)+M\left(-H_{\max }\right)\right) / 2$, where $M\left(+H_{\max }\right)$ and $M\left(-H_{\max }\right)$ are the total magnetization values of system as magnetic fields get positive and negative extrema, respectively. Figure 2 depicts the dependence of $H_{E}$ and $M_{E}$ on $K_{C}$ and $H_{F C}$.

The results indicate that when $K_{C}$ is weak $\left(K_{C} / J_{S}<\right.$ $3.0), H_{E}$ is fluctuated only in the negative value range even after cooling under strong $H_{F C}$. Once $K_{C} / J_{S}>3.0$, $H_{E}$ will change from the negative value to the positive value with the increase of $H_{F C}$. On the other hand, as shown in Fig. 2(b), no surplus magnetization exists as $K_{C}$ and $H_{F C}$ are both weak, and then with the increase of $K_{C}$ an interesting slightly negative surplus magnetization appears. Finally, when $g \mu_{B} H_{F C} / J_{S}$ is above 2.5 the surplus magnetization increases abruptly and levels off for stronger $K_{C}$ and $H_{F C}$. Generally, the surplus magnetization of system is related to the uncompensated spins in the antiferromagnet. During field cooling, the spins in the FM shell are prone to pointing to the direction parallel to $H_{F C}$. Although the nearest-neighbor spins in the AFM core at low temperature after cooling under weak fields tend to be aligning antiparallel to each other, the uncompensated spins on the surface of AFM core always exist due to the lost AFM interaction bonds. Moreover, the AFM interfacial coupling at the AFM/FM interface arranges the AFM and FM spins in the opposite directions. With the decrease of temperature through $T_{N}$, the AFM spins become frozen. Weak $K_{C}$ cannot pin the AFM spins during the hysteresis loops. However, as long as $K_{C}$ is strong enough, effect of AFM interfacial coupling on the uncompensated spins on the surface of AFM core during cooling under weak fields can be reserved in the hysteresis loops. Therefore, low-temperature frozen uncompensated spins on the surface of AFM core pointing to the opposite direction of $H_{F C}$ contribute to the appearance of slightly negative surplus magnetization.

In order to reveal the origin of phenomena for strong $H_{F C}$, typical microscopic spin configurations of nanoparticle, in which the spin in the AFM core possesses a weak or strong anisotropy, taken at selected values of fields close to the coercive fields of hysteresis loops at low temperature after cooling under strong $H_{F C}$ are given in Fig. 3 . It is found that the spins in the AFM core cannot be frozen during magnetizing when their anisotropies are weak (see Fig. 3(a)), although in the cooling process strong magnetic fields can drive all spins of nanoparticle along their directions (the results are not shown here). In other words, the effect of cooling field cannot be reserved and reflected in the hysteresis loops at low temperature to tune EB. At this point surplus magnetization of nanoparticle 

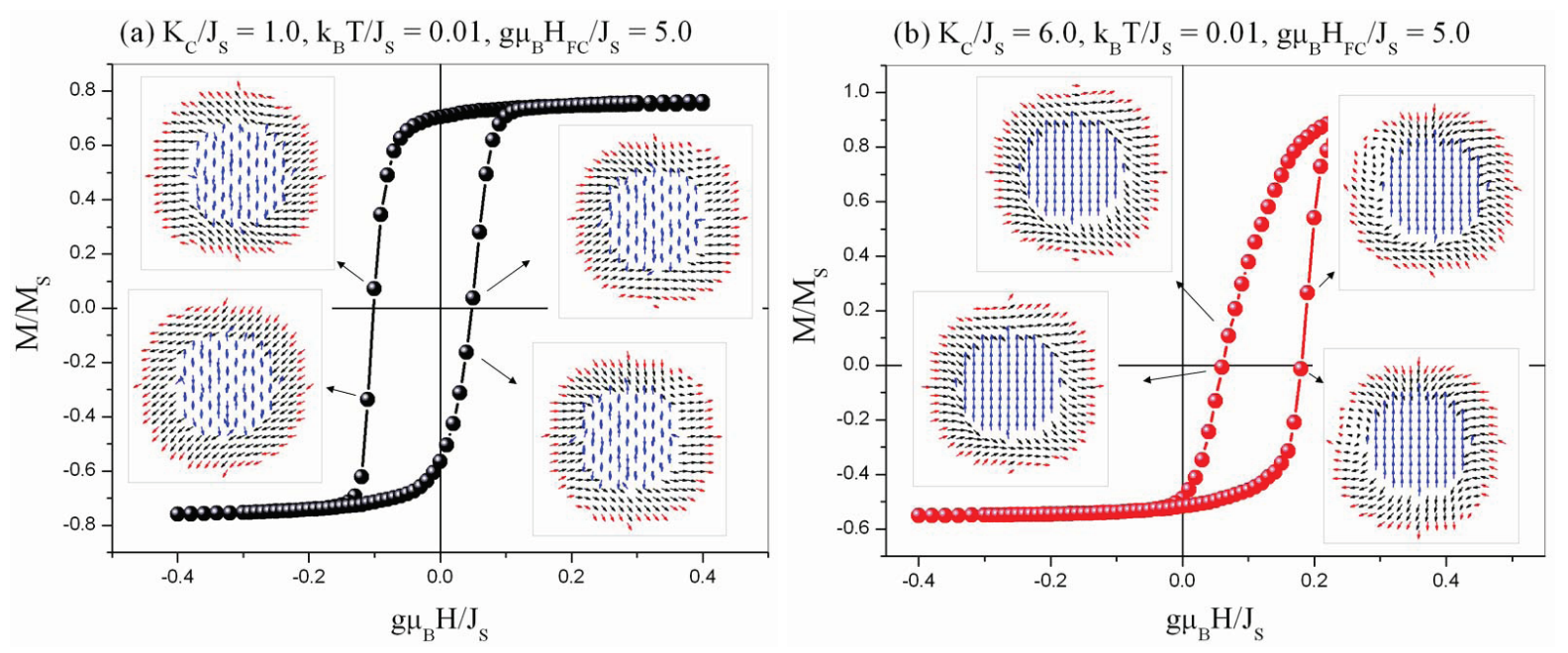

FIG. 3: Typical hysteresis loops of nanoparticles with (a) $K_{C} / J_{S}=1.0$ and (b) 6.0 at $k_{B} T / J_{S}=0.01$ after cooling under $g \mu_{B} H_{F C} / J_{S}=5.0$. Inset shows the snapshots of the microscopic spin configurations in the vertical section central plane through the symmetric axis of nanoparticle taken at selected values of fields close to the coercive fields of hysteresis loops. Red, black, and blue arrows represent the spins belonging to the core, shell, and surface of nanoparticle, respectively. $M_{S}$ is the saturated magnetization of system.

hardly appears at low temperature after cooling under different fields (see Fig. 2(b)). Moreover, the AFM core with weak anisotropy of spin can be regarded as another a soft magnet to contribute to the enhancement of coercivity (the results are also not shown in the present paper). However, because of the existence of AFM interfacial coupling, the magnetizing along different directions may produce an irreversible behavior of magnetization reversal. Thus in the nanoparticle where the spin in the AFM core possesses relatively weak anisotropies, negative EB is always observed and independent of $H_{F C}$.

On the other hand, with the increase of $K_{C}$, the pinning ability of spin in the AFM core is enhanced. $H_{F C}$ may play a decisive role in the creation of the interfacial spin configuration for EB [42], that is, different microscopic configurations of nanoparticle may be chosen at low temperature after cooling under different fields. Due to the small size of AFM core, the spins in the AFM core may even exhibit a "parallel configuration" at low temperature after cooling under relatively strong fields, as shown in Fig. 2(b). Similar results have been obtained experimentally [43] and theoretically [28, 29]. Large positive surplus magnetization will produce large positive $H_{E}$. Moreover, as shown in Fig. 3(b), in the magnetizing process two distinct magnetization reversal mechanisms are observed at both branches of hysteresis loop, namely, the reversal by coherent rotation occurs at the decreasing branch of hysteresis loop while the reversal by nucleation appears at the other side and the phenomena are evident. Actually, $H_{E}$ varies with the increase of $H_{F C}$ from the negative value to the positive value accompanying the exchanges between magnetization reversal mechanisms at both branches of hysteresis loop as reported in the earlier work [29]. However, strong $K_{C}$ which provides stable frozen configurations of nanoparticle during magnetizing at low temperature is a prerequisite to manifesting the peculiar magnetic properties.

Finally, we explicate briefly why such four magnetic states are chosen in the hysteresis loops. As a rule, only some special points of magnetic energy such as the states with $M / M_{S}=0.0$ may be taken into account. Nevertheless, during magnetizing the magnetic states change continuously with magnetic field, in other words, the evolution of the magnetic states is actually a continuous process. The scientists have found that the existence of $H_{E}$ often accompanies the distinct magnetization reversal mechanisms at both branches of hysteresis loops [44, 45]. The origin of $H_{E}$ may be illuminated by analyzing the magnetization reversal mechanisms, which are identical for all magnetic states at the same branch of a hysteresis loop. Therefore, the selection of the magnetic states in the present paper is also valid for interpreting the EB behavior.

\section{CONCLUSION}

A modified Monte Carlo Metropolis method is performed to simulate the effects of $K_{C}$ and $H_{F C}$ on $H_{E}$ and $M_{E}$ in the single composite nanoparticle with unconventional AFM (core)/FM (shell) morphology. It is found that when $K_{C}$ is weak, $H_{E}$ is present and negative as well as independent of $H_{F C}$ at low temperature after field cooling. However, once $K_{C}$ is above a critical value, $H_{E}$ will change its sign from negative to positive with the increase of $H_{F C}$, and finally level off. The phenomena are interpreted well by studying the microscopic spin configurations of nanoparticle. It is revealed that strong $K_{C}$ may determine the stabilization of frozen configurations, namely, the surplus magnetization at low temperature after field cooling to tune EB.

\section{Acknowledgments}

This study is supported by the Shenyang Applied Basic Research Foundation (Grant No. 1071199-1-00-2) of China. 
[1] R. H. Kodama, J. Magn. Magn. Mater. 200, 359 (1999).

[2] Ò. Iglesias, A. Labarta, and X. Batlle, J. Nanosci. Nanotechnol. 8, 2761 (2008).

[3] V. Skumryev, S. Stoyanov, Y. Zhang, G. Hadjipanayis, D. Givord, and J. Nogués, Nature (London) 423, 850 (2003).

[4] W. H. Meiklejohn and C. P. Bean, Phys. Rev. 102, 1413 (1956); 105, 904 (1957).

[5] J. Nogués, J. Sort, V. Langlais, V. Skumryev, S. Suriñach, J. S. Muñoz, and M. D. Baró, Phys. Rep. 422, 65 (2005).

[6] J. Nogués, D. Lederman, T. J. Moran, and I. K. Schuller, Phys. Rev. Lett. 76, 4624 (1996).

[7] S. Mangin, F. Montaigne, and A. Schuhl, Phys. Rev. B 68, 140404(R) (2003).

[8] T. L. Kirk, O. Hellwig, and E. E. Fullerton, Phys. Rev. B 65, 224426 (2002).

[9] S. Karmakar, S. Taran, E. Bose, and B. K. Chaudhuri, Phys. Rev. B 77, 144409 (2008).

[10] B. Beckmann, U. Nowak, and K. D. Usadel, Phys. Rev. Lett. 91, 187201 (2003).

[11] B. Beckmann, K. D. Usadel, and U. Nowak, Phys. Rev. B 74, 054431 (2006).

[12] J. Camarero, J. Sort, A. Hoffmann, J. M. García-Martín, B. Dieny, R. Miranda, and J. Nogués, Phys. Rev. Lett. 95, 057204 (2005).

[13] M. Vasilakaki and K. N. Trohidou, Phys. Rev. B 79, 144402 (2009)

[14] D. W. Kavich, J. H. Dickerson, S. V. Mahajan, S. A. Hasan, and J. H. Park, Phys. Rev. B 78, 174414 (2008).

[15] A. E. Berkowitz, G. F. Rodriguez, J. I. Hong, K. An, T. Hyeon, N. Agarwal, D. J. Smith, and E. E. Fullerton, Phys. Rev. B 77, 024403 (2008).

[16] J. A. Borchers, Y. Ijiri, D. M. Lind, P. G. Ivanov, R. W. Erwin, A. Qasba, S. H. Lee, K. V. O'Donovan, and D. C. Dender, Appl. Phys. Lett. 77, 4187 (2000).

[17] M. Gruyters, Phys. Rev. B 79, 134415 (2009).

[18] M. Fecioru-Morariu, S. R. Ali, C. Papusoi, M. Sperlich, and G. Güntherodt, Phys. Rev. Lett. 99, 097206 (2007).

[19] W. B. Cui, W. J. Hu, Y. J. Zhang, X. H. Liu, S. Guo, F. Yang, W. Liu, and Z. D. Zhang, J. Magn. Magn. Mater. 321, 1943 (2009).

[20] T. R. Gao, Z. Zhi, S. M. Zhou, R. Chantrell, P. Asselin, X. J. Bai, J. Du, and Z. Z. Zhang, J. Appl. Phys. 105, 053913 (2009)

[21] X. P. Qiu, D. Z. Yang, S. M. Zhou, R. Chantrell, K. O'Grady, U. Nowak, J. Du, X. J. Bai, and L. Sun, Phys. Rev. Lett. 101, 147207 (2008).

[22] J. McCord, R. Kaltofen, O. G. Schmidt, and L. Schultz, Appl. Phys. Lett. 92, 162506 (2008).

[23] D. Mauri, E. Kay, D. Scholl, and J. K. Howard, J. Appl.
Phys. 62, 2929 (1987).

[24] C. Mitsumata, A. Sakuma, and K. Fukamichi, J. Phys. Soc. Jpn. 76, 024704 (2007).

[25] A. López-Ortega, D. Tobia, E. Winkler, I. V. Golosovsky, G. Salazar-Alvarez, S. Estradé, M. Estrader, J. Sort, M. A. González, S. Suriñach, J. Arbiol, F. Peiró, R. D. Zysler, M. D. Baró, and J. Nogués, J. Am. Chem. Soc. 132, 9398 (2010).

[26] G. Salazar-Alvarez, J. Sort, S. Suriñach, M. D. Baró, and J. Nogués, J. Am. Chem. Soc. 129, 9102 (2007).

[27] I. V. Golosovsky, G. Salazar-Alvarez, A. López-Ortega, M. A. González, J. Sort, M. Estrader, S. Suriñach, M. D. Baró, and J. Nogués, Phys. Rev. Lett. 102, 247201 (2009).

[28] Y. Hu, Y. Liu, and A. Du, J. Appl. Phys. 108, 033904 (2010).

[29] Y. Hu, Y. Liu, and A. Du, Phys. Status Solidi B 247, 972 $(2010)$.

[30] Y. Hu, Y. Ma, Y. Liu, and A. Du, J Nanosci. Nanotechnol. 10, 7343 (2010).

[31] S. E. Inderhees, J. A. Borchers, K. S. Green, M. S. Kim, K. Sun, G. L. Strycker, and M. C. Aronson, Phys. Rev. Lett. 101, 117202 (2008).

[32] B. Berger, Y. Labaye, M. Tamine, and J. M. D. Coey, Phys. Rev. B 77, 104431 (2008).

[33] Y. Hu and A. Du, J. Nanosci. Nanotechnol. 9, 5829 (2009).

[34] L. Néel, J. Phys. Radium 15, 255 (1954).

[35] H. F. Du and A. Du, J. Appl. Phys. 99, 104306 (2006).

[36] Y. Hu and A. Du, J. Magn. Magn. Mater. 322, 844 (2010).

[37] J. Sort, A. Popa, B. Rodmacq, and B. Dieny, Phys. Rev. B 70, 174431 (2004).

[38] G. Vallejo-Fernandez, L. E. Fernandez-Outon, and K. O'Grady, Appl. Phys. Lett. 91, 212503 (2007).

[39] J. Keller, P. Miltényi, B. Beschoten, G. Güntherodt, U. Nowak, and K. D. Usadel, Phys. Rev. B 66, 014431 (2002).

[40] J. Geshev, J. Magn. Magn. Mater. 320, 600 (2008).

[41] Y. Tang, Y. Sun, and Z. Cheng, Phys. Rev. B 73, 174419 (2006).

[42] S. Guo, X. H. Liu, W. B. Cui, W. Liu, X. G. Zhao, D. Li, and Z. D. Zhang, J. Appl. Phys. 105, 064702 (2009).

[43] R. Morales, Z. P. Li, J. Olamit, K. Liu, J. M. Alameda, and I. K. Schuller, Phys. Rev. Lett. 102, 097201 (2009).

[44] J. McCord, R. Schäfer, R. Mattheis, and K. U. Barholz, J. Appl. Phys. 93, 5491 (2003).

[45] X. Ji, A. B. Pakhomov, and K. M. Krishnan, J. Appl. Phys. 101, 09E507 (2007). 